\title{
HIGHLIGHTS
}

TRANSPLANTATION

\section{Nephrectomy of a failed allograft decreases mortality}

The number of patients who return to long-term dialysis after kidney transplantation is growing, and these patients may be at high risk of death. Juan Carlos Ayus and colleagues have now found, however, that removal of the failed allograft is associated with improved survival in such patients returning to long-term dialysis.

\section{According to Ayus et al., no} consensus exists on how to best manage patients with a failed renal transplant... 77

According to Ayus et al., no consensus exists on how to best manage patients with a failed renal transplant, and no randomized clinical trial of renal transplant recipients on long-term dialysis has compared the effect of removing the failed allograft with leaving it in place. In the USA, allograft nephrectomy is usually performed in patients with hyperacute allograft rejection, hematuria, serious infection, graft thrombosis, or graft intolerance syndrome.
Ayus et al. identified in the US Renal Data System database 10,951 renal transplant recipients who started longterm dialysis treatment between 1994 and 2004. Of these patients, 3,451 (31.5\%) underwent allograft nephrectomy during follow-up. After a mean follow-up of 2.93 years, 1,106 transplant recipients in the nephrectomy group and 2,679 transplant recipients in the non-nephrectomy group had died. The researchers adjusted these results to take into account the different sociodemographic characteristics of transplant recipients and donors, recipient comorbidities, incident clinical conditions associated with allograft nephrectomy, and recipient characteristics that qualified the patient for allograft nephrectomy.

After adjustment for these confounders, the risk of all-cause mortality was $32 \%$ lower in transplant recipients with a failed allograft who underwent transplant nephrectomy than in those who did not undergo nephrectomy. Furthermore, patients who underwent allograft nephrectomy were more likely to receive a second transplant during follow-up than patients who did not undergo nephrectomy. In addition, the rate of perioperative mortality associated with allograft nephrectomy was much lower than expected (1.5\% versus $6-37 \%$ reported in the literature). Ayus et al. conclude that a future randomized trial should evaluate the feasibility of allograft nephrectomy as a routine treatment in patients on dialysis with a failed renal allograft.

Ayus comments that the propensity score approach employed in the current study is the next best option after a randomized trial for achieving clinically reliable data. He also hypothesizes that the increased risk of mortality observed in patients with a failed renal allograft who did not undergo nephrectomy might be a result of the failed graft causing chronic inflammation, a major risk factor for cardiovascular mortality in patients on dialysis. Ayus is currently investigating the mechanisms by which removal of a failed renal allograft might decrease the risk of mortality.

\section{Baldo Lucchese}

Original article Ayus, J. C. et al. Transplant nephrectomy improves survival following a failed renal allograft. J. Am. Soc. Nephrol. 21, 374-380 (2010) 